\title{
Effect of front TCO on the performance of rear-junction silicon heterojunction solar cells: Insights from simulations and experiments
}

\author{
Alexandros Cruz ${ }^{\mathrm{a}}$, Er-Chien Wang ${ }^{\mathrm{a}}$, Anna B. Morales Vilches ${ }^{\mathrm{a}}$, Daniel Meza ${ }^{\mathrm{b}}$, Sebastian Neubert ${ }^{\mathrm{a}}$, \\ Bernd Szyszka ${ }^{c}$, Rutger Schlatmann ${ }^{\mathrm{a}}$, Bernd Stannowski ${ }^{\mathrm{a}}$ \\ ${ }^{a}$ Helmholtz-Zentrum Berlin, PVcomB, Schwarzschildstr. 3, 12489 Berlin, Germany \\ ${ }^{b}$ Helmholtz-Zentrum Berlin, Institute for Silicon Photovoltaics, Kekuléstraße 5, 12489 Berlin, Germany \\ ${ }^{c}$ Technische Universität Berlin, Einsteinufer 25, 10587 Berlin, Germany
}

alexandros.cruz@helmholtz-berlin.de,er-chien.wang@helmholtz-berlin.de, ana.morales_vilches@helmholtz-berlin.de, daniel.meza@helmholtz-berlin.de, sebastian.neubert@helmholtz-berlin.de, bernd.szyszka@@tu-berlin.de, rutger.schlatmann@helmholtzberlin.de, bernd.stannowski@helmholtz-berlin.de

Corresponding Author: Alexandros Cruz ; alexandros.cruz@helmholtz-berlin.de

\begin{abstract}
In this study we make a detailed comparison between indium tin oxide (ITO), aluminum-doped zinc oxide ( $\mathrm{ZnO}: \mathrm{Al}$ ) and hydrogenated indium oxide (IO:H) when applied on the illuminated side of rear-junction silicon heterojunction (SHJ) solar cells. ITO being the state of the art material for this application, ZnO:Al being an attractive substitute due to its cost effectiveness and IO:H being a transparent conductive oxide (TCO) with high-mobility and excellent optical properties. Through numerical simulations, the optically optimal thicknesses for a double layer anti-reflective coating system, consisting of the respective TCO and amorphous silicon oxide (a-SiO $\left.{ }_{2}\right)$ capping layers are defined. Through two-dimensional electrical simulations, we present a comparison between front-junction and rear-junction devices to show the behavior of series resistance $\left(R_{s}\right)$ in dependence of the TCO sheet resistance $\left(R_{s h}\right)$ and the device effective lifetime $\left(\tau_{e f f}\right)$. The study indicates that there is a $\tau_{\text {eff }}$ dependent critical TCO $R_{s h}$ value, above which, the rear-junction device will become advantageous over the front-junction design in terms of $R_{s}$. Solar cells with the respective layers are analyzed. We show that a thinner TCO optimized layer will result in a benefit in cell performance when implementing a double layer anti-reflective coating. We conclude that for a highest efficiency solar cell performance, a high mobility TCO, like IO:H, is required as the device simulations show. However, the rear-junction solar cell design permits the implementation of a lower conductive TCO in the example of the cost-effective $\mathrm{ZnO}$ :Al with comparable performance to the ITO, opening the possibility for substitution in mass production.
\end{abstract}

Keywords: Transparent conductive oxide, Silicon heterojunction, Rear-junction, Sheet resistance, Anti-reflective coating, Series resistance

\section{Introduction}

Silicon heterojunction (SHJ) solar cells have gained significant interest in the past years due to their high performance, with record efficiencies of $25.1 \%$ and $26.7 \%$ for two-side and all-back contacted cells, respectively [1][2][3]. The main driver for these achievements has been the excellent passivation of the crystalline silicon (c-Si) wafer by thin hydrogenated amorphous silicon (a$\mathrm{Si}: \mathrm{H})$ contacts for electrons and holes leading to high open circuit voltages $\left(V_{o c}\right)$. Unlike silicon solar cells with diffused (homo)junction for which the carriers are transported to the metal contacts within the wafer, the SHJ cell concept requires transparent conductive oxide (TCO) layers on top of the a-Si:H contacts for efficient lateral carrier transport towards the metal contacts. The development of TCO materials with high-mobility and low parasitic absorption facilitated short circuit current densities $\left(J_{s c}\right)$ of over $40 \mathrm{~mA} / \mathrm{cm}^{2}$ with fill factors $(F F)$ well above $80 \%$ [4].

In today's crystalline silicon solar cells the (homo)junction is usually placed at the front (illuminated) side of the wafer for various reasons, e.g. to support the collection of minority carriers with a shorter average path length to the illuminated side. For the SHJ, however, the design rules are different. This is due to the symmetric design of electron and hole contacts as well as the use of monocrystalline silicon wafers (usually n-type) with very high carrier lifetimes, hence long diffusion lengths. Placing the junction on the rear side allows the wafer to support the front side TCO to laterally transport the majority carriers and, in turn, relaxes the requirements on the conductivity of the front TCO. This was shown and discussed in depth by Bivour et al. [5]. Here we present a detailed comparison of different front-TCOs and its effect on the performance of SHJ solar cells both by simulations and in experimental cells. The investigated TCOs are indium tin oxide (ITO), aluminum-doped zinc oxide (ZnO:Al) and hydrogenated indium oxide (IO:H). ITO has good opto-electrical properties and long-term stability [6], which is the reason for its widespread use for optoelectronic applications and has become a standard material for SHJ solar cells. $\mathrm{ZnO} \mathrm{Al}$ is a natively 
polycrystalline material with lower electronic quality than ITO when deposited at low temperatures $\left(<200^{\circ} \mathrm{C}\right)$ and as thin layer $(<100 \mathrm{~nm})$. The abundance of zinc in the earth's crust, however, makes it an attractive low-cost substitute for indium-based TCOs [7]. IO:H on the other hand is a TCO with carrier mobility over $100 \mathrm{~cm}^{2} / \mathrm{Vs}$ and low parasitic absorption [8]. In our study we investigated two cases: firstly, a TCO thickness of $75 \mathrm{~nm}$, typical for best anti-reflection (AR) effect with solar irradiation (AM1.5g) on silicon [9] and secondly, thinner optimized TCO layers for reduced parasitic absorption. In both cases we added an $\mathrm{a}-\mathrm{SiO}_{2}$ layer as second AR layer on top to further reduce reflection losses [10]-[14]. For the analysis we carried out optical simulations with the Matlab-based one-dimensional program GenPro4 [15]. By adapting the TCO and the a-SiO 2 thicknesses, we optimized this layer system for highest photocurrent density of the SHJ device for each of the three investigated materials. Once the optimal thicknesses were known, electrical simulations were carried out with the two-dimensional program Quokka2 [16] to rate the total opto-electrical device performance, which is then compared to the performance of experimental cells. As a result we show, that the IO:H leads to highest efficiencies according to simulations. However, thanks to the rear-junction cell design the use of $\mathrm{ZnO}: \mathrm{Al}$ as a front-TCO is possible without having a significant efficiency penalty.

\section{Materials and Methods \\ 2.1. TCO Deposition and Characterization}

TCO layers with thicknesses of $105 \pm 10 \mathrm{~nm}$ measured with a Dektak profilometer were deposited on $1.1 \mathrm{~mm}$ thick Corning Eagle glass in an in-line DC magnetron sputtering system from Leybold Optics (A600V7). Such TCO layers have an approx. thickness of $75 \mathrm{~nm}$ when deposited on textured wafers as described in section 2.2. ITO layers were sputtered from a planar 97:3 $\mathrm{In}_{2} \mathrm{O}_{3}: \mathrm{SnO}_{2}$ target, $\mathrm{ZnO}: \mathrm{Al}$ from a rotatable 99:1 $\mathrm{ZnO}: \mathrm{Al}_{2} \mathrm{O}_{3}$ target, and IO:H from a ceramic planar $\mathrm{In}_{2} \mathrm{O}_{3}$ target. ITO and $\mathrm{ZnO}: \mathrm{Al}$ were deposited at an approximate substrate temperature of $150^{\circ} \mathrm{C}$ with oxygen flow ratios $\mathrm{r}\left(\mathrm{O}_{2}\right)=\mathrm{q}\left(\mathrm{O}_{2}\right) / \mathrm{q}\left(\mathrm{Ar}+\mathrm{O}_{2}\right)$ of $2.4 \%$ and $0.48 \%$, respectively. IO:H was deposited without intentional heating at an oxygen flow ratio of $2.0 \%$ and with an introduction of water vapor resulting in $1.7 \times 10^{-6} \mathrm{mbar}$ partial pressure. The depositions were carried out at a pressure of 2.6, 3.6 and $4.4 \mu \mathrm{bar}$ for ITO, $\mathrm{ZnO}: \mathrm{Al}$ and IO:H respectively. The system base pressure was of approx. $0.4 \mu \mathrm{bar}$ in the sputtering chamber before any intentional gas inlet. The IO:H samples were post-deposition annealed in vacuum at $180^{\circ} \mathrm{C}$ for $1 \mathrm{~h}$ for crystallization.

Charge carrier mobility, $\mu_{\text {Hall }}$, and concentration, $N_{\mathrm{e}}$, were determined for TCO layers on glass by Hall measurements with an Ecopia HMS 3000 system applying the van der Pauw method at room temperature. The optical characterization of the TCOs was carried out with a Perkin Elmer Lambda 1050 spectrophotometer equipped with a $150 \mathrm{~mm}$ integrating sphere. Refractive indices $n$ and extinction coefficients $k$ of TCO samples deposited on glass and on flat silicon substrates were extracted from spectrophotometer and spectroscopic ellipsometry measurements by fitting to a Drude-Tauc-Lorentz model [17].

\subsection{Solar Cells Preparation and Characterization}

For solar cells preparation, n-type Czochralski (CZ) silicon wafers (c-Si) with $5 \Omega \mathrm{cm}$ resistivity were used. The as-cut wafers were wet-etched to remove the saw damage. Its surfaces were then chemically textured in $\mathrm{KOH}$ to obtain random pyramids with heights in the range of $2-4 \mu \mathrm{m}$ with $<111>$ oriented facets and resulting in $125-\mu \mathrm{m}$ thick wafers. After RCA cleaning [18] finalized with a dip in a $1 \%$ diluted hydrofluoric acid solution, intrinsic and doped silicon layers were deposited by plasma enhanced chemical vapor deposition (PECVD) in an AKT1600 cluster tool from Applied Materials with a parallel electrode configuration operated at $13.56 \mathrm{MHz}$. An i/p a-Si:H stack was deposited on the rear side to form the hole contact (junction). At the front side an electron contact was formed by depositing an i-a-Si:H with an n-type nanocrystalline silicon (nc-Si:H) layer on top. Further details of the silicon thin-films used can be found elsewhere [19].

Monofacial solar cells were prepared by varying the front TCO layers and with a $\mathrm{ZnO}: \mathrm{Al} / \mathrm{silver}$ rear reflector. These layers were sputtered through aligned shadow masks on both sides of the wafer to define 4- $\mathrm{cm}^{2}$ size cells and transfer length method (TLM) structures. Only the IO:H coated solar cells, underwent a thermal annealing process of $1 \mathrm{~h}$ at $185^{\circ} \mathrm{C}$ under vacuum in order to solid-phase crystallize the TCO. A silver grid with $1670 \mu \mathrm{m}$ pitch and $50 \mu \mathrm{m}$ finger thickness resulting in $\sim 3 \%$ total shading was screen printed on the front TCO and subsequently cured at $210^{\circ} \mathrm{C}$ for 15 minutes on a hot-plate under atmospheric conditions. Finally, a PECVD a-SiO 2 layer was deposited on top of the finished devices as second anti-reflective coating. The process was carried out at gas flows of 4.5, 900 and $500 \mathrm{sccm}$ for $\mathrm{SiH}_{4}, \mathrm{H}_{2}, \mathrm{~N}_{2} \mathrm{O}$, respectively, at $185^{\circ} \mathrm{C}$ and at $30 \mathrm{~W}$.

The solar cells were characterized using current voltage $(J-V)$ measurements in the dark and under an AM1.5G spectrum at standard test conditions in a Wacom WXS-155S-L2 dual source class AAA+ sun simulator. Resistance measurements on the TLM structures to calculate TCO-silver contact resistivity and TCO sheet resistance $\left(R_{\text {sh }}\right)$ on devices were carried out. To derive the front TCO $R_{\mathrm{sh}}$ from a rear-junction device from TLM structures we used a parallel resistance model as proposed by Bivour et al. [5]. According to $R_{s h T C O}=1 /\left(1 / R_{s h T L M}-1 / R_{\text {sh Wafer }}\right)$ with $R_{s h ~ T C O}$ being the TCO sheet resistance, $R_{s h}$ TLM the sheet resistance measured via TLM structures and $R_{s h}$ wafer the sheet resistance of the wafer. The wafer sheet resistance was kept constant at $400 \Omega$ calculated from the nominal resistivity of the wafers of $5 \Omega \mathrm{cm}$ divided by its thickness nominally of $125 \mu \mathrm{m}$. 
Series Resistance $\left(R_{s}\right)$ values of the solar cells were determined from the dark to light $J-V$ curve comparison after Pysch et al. [20].

\section{Results and Discussion}

\subsection{TCO Layer Properties}

\subsubsection{Electrical Properties}

Sputtered ITO exhibits $\mu_{\text {Hall }}>30 \mathrm{~cm}^{2} / \mathrm{Vs}$ in a broad range of carrier concentrations from 1 to $4 \times 10^{20} \mathrm{~cm}^{-3}$ even for thin layers $<100 \mathrm{~nm}$ [21]. ZnO:Al layers, in contrast, reach these values only when deposited as several $100 \mathrm{~nm}$ thick layers typically at temperatures well above $200^{\circ} \mathrm{C}$ so that large crystal grains can be formed during growth. With the restrictions given by the SHJ solar cell processing, namely $<100 \mathrm{~nm}$ TCO thickness and temperatures $<200^{\circ} \mathrm{C}$, the $\mathrm{ZnO}$ :Al properties worsen, i.e. the $100-\mathrm{nm}$ thick ZnO:Al layers here reach mobilities of only $10-20 \mathrm{~cm}^{2} / \mathrm{Vs}$, limited by the small-grained polycrystalline growth [22]. In comparison, IO:H is a high-mobility TCO reaching $\mu_{\text {Hall }}>100 \mathrm{~cm}^{2} / \mathrm{Vs}$ even for very thin layers processed below $200^{\circ} \mathrm{C}$. Koida et al. demonstrated that these remarkable values are due to solid-phase crystallization upon post-deposition annealing leading to lateral grain sizes up to $400 \mathrm{~nm}$ with relaxed grain boundaries that are formed from the as-deposited amorphous material. During sputtering the crystal growth is suppressed by adding water $\left(\mathrm{H}_{2} \mathrm{O}\right)$ vapor to the argon process gas [23].

We optimized the deposition parameters for $105 \pm 10 \mathrm{~nm}$ thick TCO layers on glass towards lowest carrier concentration possible in order to minimize the free-carrier absorption (FCA), yet still showing high mobility values. As we can see in Figure 1 the carrier concentration for the three TCOs investigated, remains within a narrow range of $2 \pm 0.5 \times 10^{20} \mathrm{~cm}^{-3}$, hence the difference in conductivity between them is dominated by their $\mu_{\text {Hall }}$. For the TCOs implemented in devices the values for $\mu_{\text {Hall }}$ are around 35 , 20 , and $100 \mathrm{~cm}^{2} / \mathrm{Vs}$, for ITO, ZnO:Al, and IO:H, respectively, with corresponding $R_{\mathrm{sh}}$ of $70 \pm 10 \Omega, 190 \pm 20 \Omega$, and $30 \pm 10 \Omega$. For simulations we calculated the sheet resistance from the equation $R_{\mathrm{sh}}=\rho / t$ where $\rho$ is the specific resistivity and $t$ is the thickness of the material. Depending on the type of TCO the value for $\rho$ might vary with $t$ as well as when being deposited on textured silicon wafers instead of glass. These effects, however, were neglected in our calculations for simplification. The contribution of the resistive losses related to the electrical properties of the TCOs and when varying the thickness of the respective materials on devices, was calculated with the Quokka2 program and is discussed in the electrical simulations section 3.2.2.

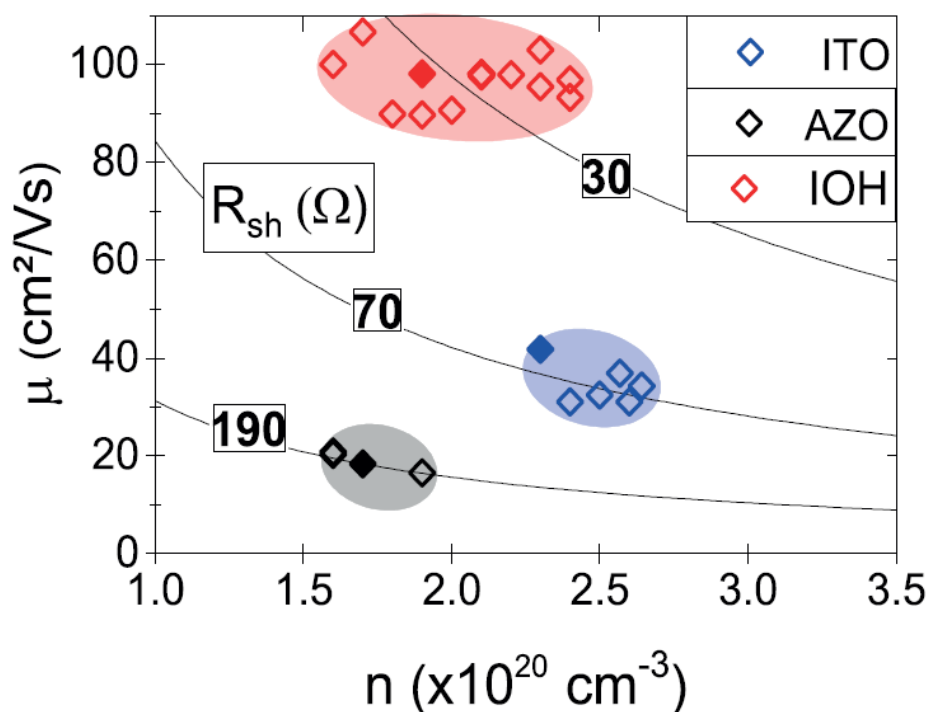

Figure 1. Mobility vs carrier concentration of $105 \pm 10 \mathrm{~nm}$ thick ITO, $\mathrm{ZnO}$ :Al and IO:H layers on glass. The colored ellipses highlight the range of TCO properties applied on SHJ experimental solar cells. The filled symbols correspond to layers optically analyzed. The sheet resistance is calculated and plotted for $105 \mathrm{~nm}$ TCO thickness.

\subsubsection{Optical Properties}


To evaluate the optical properties of the different TCOs on glass, total reflection $R$ and total transmission $T$ spectra were measured for wavelengths from 300 to $1200 \mathrm{~nm}$. Figure 2 shows absorption spectra calculated as $A=1-T$ - R. Additionally, the simulated SHJ solar cell generated current density of our standard cell stack with an assumed TCO extinction coefficient $k=0$ is shown to highlight the range where the parasitic absorption of the TCOs is most relevant. In the near infrared range $>700 \mathrm{~nm}$ the ITO has higher absorption than the $\mathrm{ZnO}: \mathrm{Al}$ and the $\mathrm{IO}: \mathrm{H}$ mostly due to its higher carrier concentration resulting in free carrier absorption (FCA). This trend continues towards shorter wavelengths where the IO: $\mathrm{H}$ and the $\mathrm{ZnO}: \mathrm{Al}$ still have a lower subbandgap absorption than the ITO. This sub-bandgap absorption is usually ascribed to defects, such as point defects, dislocations, or grain boundaries [24]. Here, the IO:H shows noticeable lower absorption than both other materials by combining a high optical bandgap, and a low sub-bandgap absorption. Furthermore the high mobility of the IO:H helps suppress the FCA [25]. At a value around $400 \mathrm{~nm}$ the $\mathrm{ZnO}: \mathrm{Al}$ suffers from the onset of the fundamental absorption due to its smaller optical bandgap of around 3.6 $\mathrm{eV}$ in comparison to $4.0 \mathrm{eV}$ for the indium-based TCOs. In total, this makes IO:H the most transparent material in the relevant range followed by the $\mathrm{ZnO}: \mathrm{Al}$. For thinner layers, as we see in the example of the $\mathrm{IO}: \mathrm{H}$ with $55 \pm 5 \mathrm{~nm}$ thickness as the dashed line in Figure 2, the absorption can be further reduced.

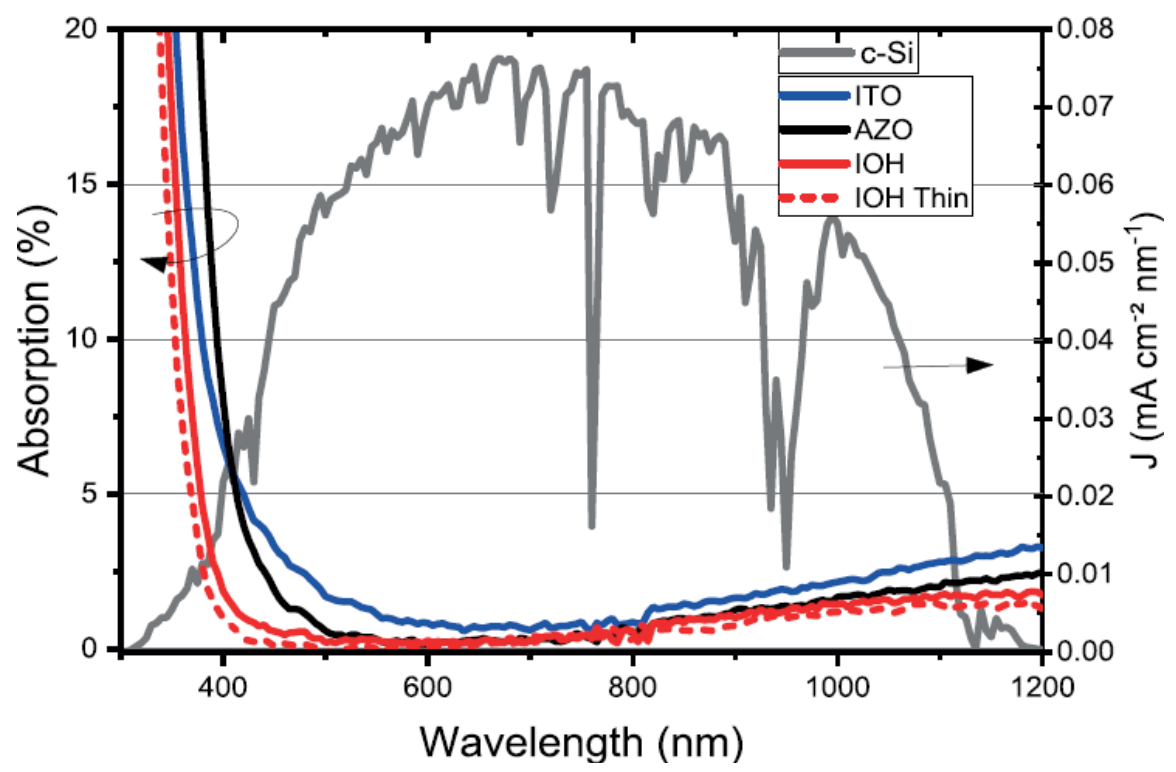

Figure 2. Optical absorption spectra of selected 105 $\pm 10 \mathrm{~nm}$ ITO, ZnO:Al and IO:H layers on glass and generated current density per wavelength of a standard SHJ solar cell from GenPro4 simulations. The dashed line is for a thinner IO:H $55 \pm 5$ nm layer.

\subsection{Device Simulations}

\subsubsection{Optical Simulations}

To quantify the optical performance of the TCO materials when integrated in SHJ cell in terms of $J_{\mathrm{sc}}$, simulations with the Matlab-based program GenPro4 [15] were carried out for the complete cell stack. For this purpose, the TCO thickness as well as the one of the $\mathrm{a}-\mathrm{SiO}_{2}$ capping layer were varied. GenPro4 uses a ray-tracing model and the net-radiation method to calculate the interface reflectance, interface transmittance and layer absorption for each component in the material stack [15].

From the simulations the optimal double layer anti-reflective coating thicknesses for the solar cells were calculated to be considered for the electrical calculations in section 3.2.2. and to define the layer thicknesses for the experimental solar cells. It is known that the electrical properties of the TCOs change when deposited on hydrogenated silicon thin-films and when hydrogenated layers are then afterwards deposited on top of them [12][26]. This effect is expected to have an impact on the optical properties of the materials as well. To our knowledge, no detailed study on the variation of the optical properties of the TCO due to adjacent hydrogenated layers for SHJ solar cells has been published and this effect would be worth of detailed investigation and comparison. For this paper, we define the trends for optimal thicknesses of the layers considering the optical properties extracted from layers on glass and for fixed optical constants throughout the thickness variations.

In Figure 3 we display the current density loss due to parasitic absorption in the TCO $J_{A b s} T C O$ in dependence of the material thickness for each TCO for a solar cell stack without a- $\mathrm{SiO}_{2}$ second anti-reflective layer. 
Higher current gain will be obtained for the higher absorptive TCO layers when lowering the material thickness. Taking a $40 \mathrm{~nm}$ TCO thickness for comparison to the $75 \mathrm{~nm}$ standard thickness reference point, the IO:H gain is of $0.2 \mathrm{~mA} / \mathrm{cm}^{2}$ whereas the ITO and AZO thinning results in 0.4 and $0.7 \mathrm{~mA} / \mathrm{cm}^{2}$ gain, respectively.

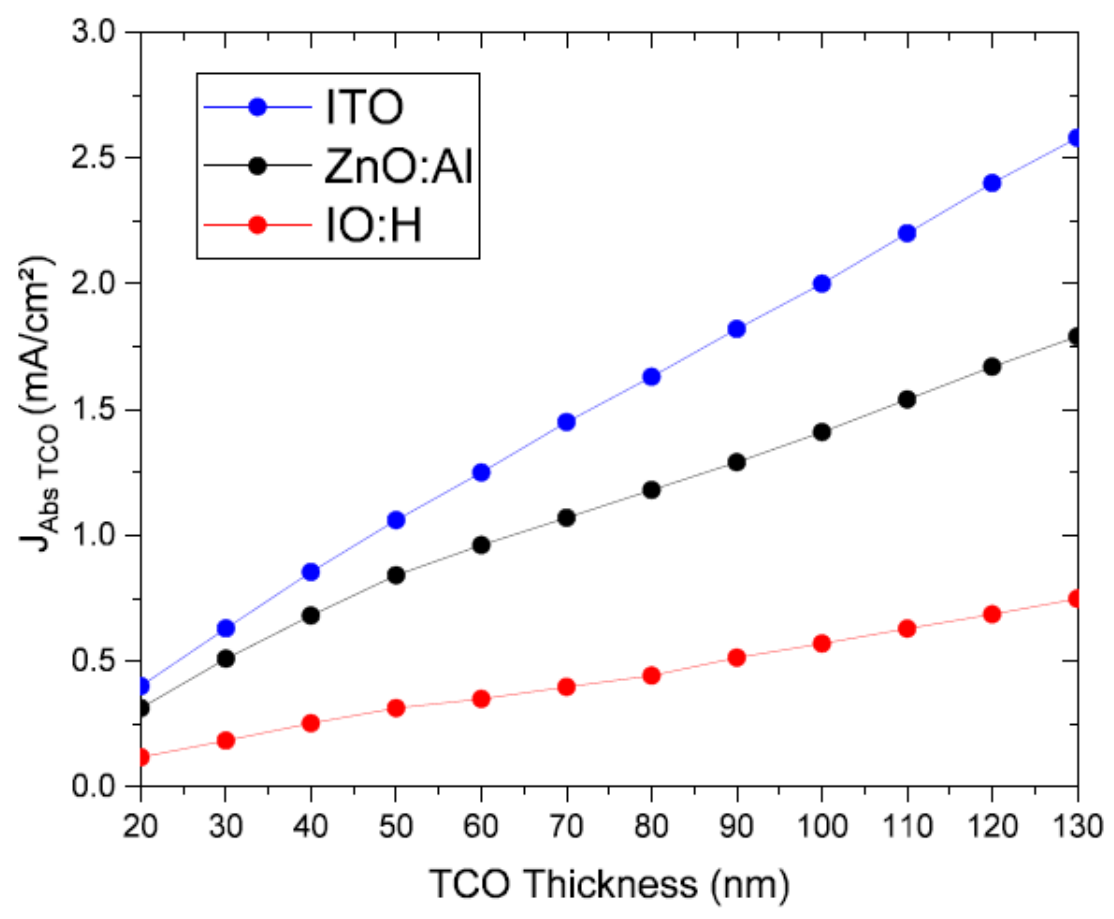

Figure 3. Current density loss due to parasitic absorption in the TCO $J_{A b s} T C O$ in dependence of the material thickness for a solar cell stack without a-SiO 2 second anti-reflective layer.

In the top row of Figure 4 we see the device reflection loss $J_{\text {Reflection }}$ color-maps for the different TCOs. The double layer antireflection coating optimum is positioned at slight lower TCO thicknesses than $75 \mathrm{~nm}$. Further reducing the TCO thickness from these points will present a trade-off between the TCO absorption decrease and the device reflection losses. A device with a more absorptive TCO will generate its maximum current density $J_{\text {Generated }}$ with thinner TCO layers further away from its anti-reflective optimum. We confirm this in the lower row of Figure 4 where $J_{\text {Generated }}$ values for the devices at standard $75 \mathrm{~nm}$ thickness and at the double layer anti-reflective optimum are noted as calculated from simulations for our solar cell material stack. The optimum thicknesses of the TCO layers are extracted being of 40, 40 and $55 \mathrm{~nm}$ for ITO, $\mathrm{ZnO}$ :Al and IO:H, respectively. A decreased TCO thickness will result in a higher $R_{\mathrm{sh}}$ and can increase the device's series resistance $R_{s}$. Therefore, in the next section we quantify the $R_{s}$ losses in the cell by means of electrical device simulations. 

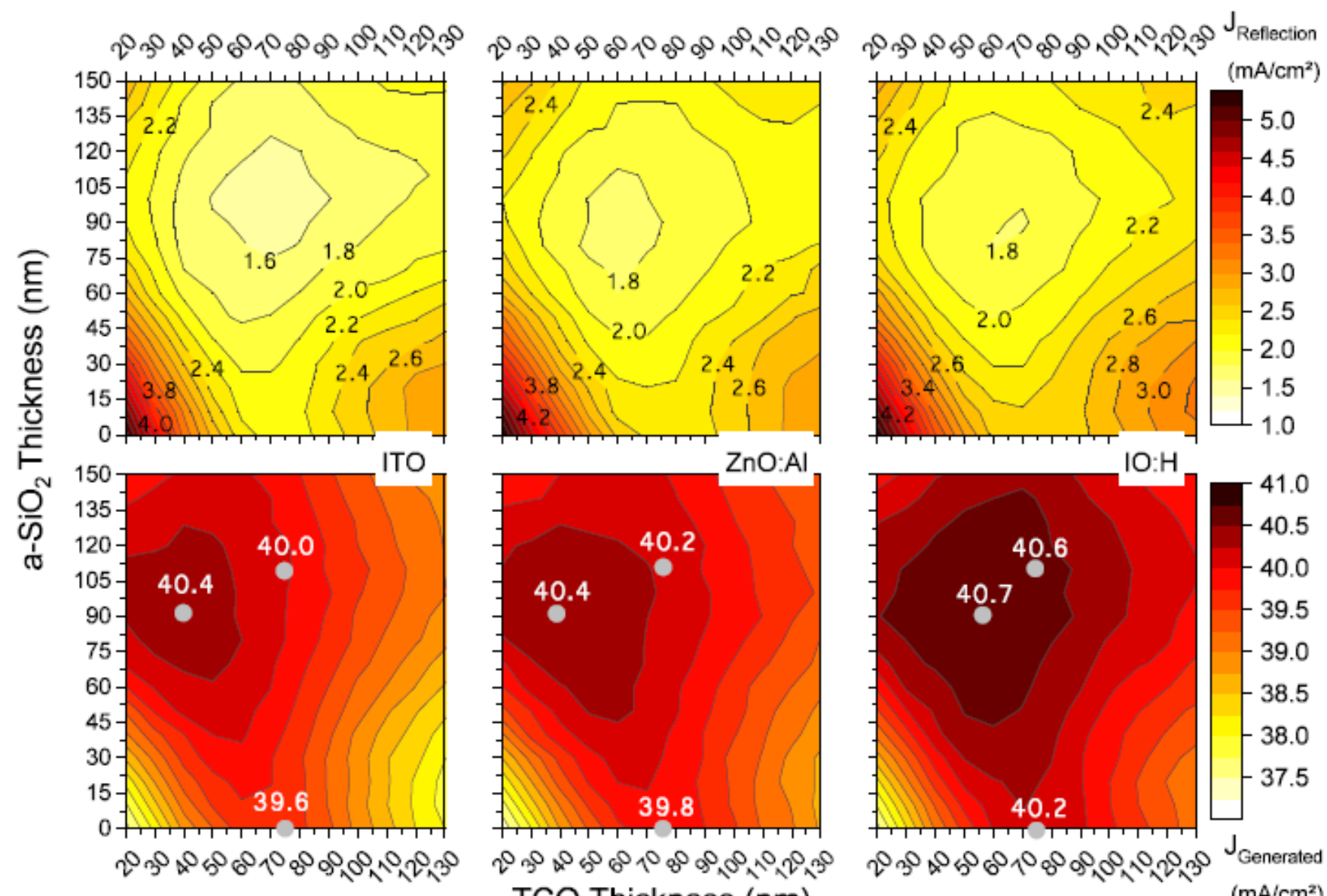

TCO Thickness $(\mathrm{nm})$

$\left(\mathrm{mA} / \mathrm{cm}^{2}\right)$

Figure 4. Top row: Colormaps of current density loss due to device reflection $J_{\text {Reflection}}$. Bottom row: Colormaps of current density generated $J_{\text {Generated }}$ in the device. For all graphs the TCO (ITO, ZnO:Al and IO:H) and the a-SiO 2 second ARC thickness on simulated silicon heterojunction solar cells are varied. Generated current in the silicon absorber $J_{\text {Generated }}$ values for $75 \mathrm{~nm}$ and optimal TCO thickness with and without a-SiO 2 optimal layers are noted.

\subsubsection{Electrical Simulations}

To simulate the electrical solar cell parameters, in particular to quantify the resistive transport losses in the wafer and in the different TCOs, Quokka2 was used, which facilitates simulations in two dimensions [16]. The geometry and material properties of our standard solar cell with $1670 \mu \mathrm{m}$ grid-finger pitch (= 12 fingers) as described in the experimental methods section 2.2. was used. For the n-type silicon wafer the Klaassen [27] model was applied to describe electrons and holes mobility. Auger recombination was modelled following Richter's approach [28]. The radiative recombination coefficient was set to $4.73 \times 10^{-15} \mathrm{~cm}^{-}$ ${ }^{3}$ according to Trupke et al. [29]. The bulk lifetime was varied from 500 to $9000 \mu \mathrm{s}$. For the rear $\mathrm{ZnO}$ :Al contacting layer a $R_{s h}=$ $200 \Omega$ was used. A dark saturation current density $J_{0}=5.5 \mathrm{fA} / \mathrm{cm}^{2}$ and recombination current density of $J_{02}=2.0 \mathrm{nA} / \mathrm{cm}^{2}$ were determined from the two-diode model fit of our standard solar cell $J$ - $V$ curve and were introduced and assumed equally distributed for the rear and front side of the device. The $R_{s h}$ of the front TCO was varied linearly with the TCO thicknesses. A geometry independent contact resistivity $\rho_{c}=3 \mathrm{~m} \Omega \mathrm{cm}^{2}$ of the metal-TCO interface was determined by the TLM measurements [30] and introduced to the simulation. The current generation was assumed to occur at the surface of the device, with a steady value at $40.5 \mathrm{~mA} / \mathrm{cm}^{2}$ for an AM1.5 solar spectrum. A lumped external $R_{s}$ of $0.4 \Omega \mathrm{cm}^{2}$ was added to account for all remaining $R_{s}$ contributions, i.e. the metal contact transport and the contact resistances at the remaining device interfaces. To calculate this value 
we measured line resistivity of the screen-printed silver and measured the geometry of the grid fingers with a confocal microscope. For the TCO-Si contact resistivity, the $R_{s}$ contribution was calculated by comparing the sum of all known contributions to the experimental value calculated from the light to dark $J$ - $V$ curves comparison.

Figure 5 shows the $R_{s}$ calculated from the resistive power loss at maximum power point, as a function of the front TCO's $R_{s h}$ and for two different effective carrier lifetimes $\tau_{\text {eff }}$ of the solar cells. One "low" at $400 \mu$ s and one in the range of the ones calculated for our experimental devices, which lies at $1750 \mu \mathrm{s}$ and is hereafter referred to as "nominal".

The average minority excess carrier density at operating point was calculated to be of $0.55 \pm 0.05 \times 10^{15} \mathrm{~cm}^{-3}$ and $2.1 \pm 0.1 \times 10^{15} \mathrm{~cm}^{-3}$ for the low and nominal $\tau_{e f f}$ defined, respectively.

In Figure 5 at a $0 \Omega R_{s h}$ TCO value the $R_{s}$ consists exclusively of the external lumped $R_{s}$ value of $0.4 \Omega \mathrm{cm}^{2}$ plus the $R_{s}$ accounting for the vertical charge carrier transport, since the lateral transport to the metal-grid will be carried out by the ideally conductive TCO without losses. We see from the simulations that the clearly higher $R_{s}$ increase for the rear-junction in comparison to the front-junction device when $\tau_{e f f}$ is decreased, is mainly dominated by the less conductive holes transport to the rear selective contact. For the front-junction device for which the electrons are the ones travelling to the rear contact, the $\tau_{\text {eff }}$ decrease will barely increase the $R_{s}$ at this point.

When increasing the TCO $R_{s h}$, lateral transport losses will arise. We can see a clearly higher $R_{s}$ increment with increasing $R_{s h}$ for the front-junction device since the TCO is partially electrically separated from the wafer by the pn depletion region for this case. Still, some of the lateral transport will take part in the wafer for the front-junction device as the TCO $R_{s h}$ increases and currentcrowding at the TCO-Si interface forces the lateral charge carrier flow within the wafer. For the rear-junction device, the $R_{s}$ increase due to the lateral transport is less pronounced, since the TCO and the wafer are electrically coupled as discussed by Bivour et al. [5].

Considering the solar cell type and the behavior of the $R_{s}$ in dependence of the TCO $R_{s h}$ for the nominal $\tau_{e f f}$, we see that for higher resistive TCOs the rear-junction design is clearly advantageous. Below $R_{s h} \approx 100 \Omega$, however, the front-junction device shows lower $R_{s}$. This is even more significant for the low $\tau_{\text {eff }}$ where the front- outperforms the rear-junction design in terms of $R_{s}$ for TCOs of up to $R_{\mathrm{sh}}=300 \Omega$. This behavior highlights the importance of considering the effect of the TCO $R_{s h}$ in combination with the $\tau_{\text {eff }}$ of the device when deciding on the design between a front-junction and a rear-junction device.

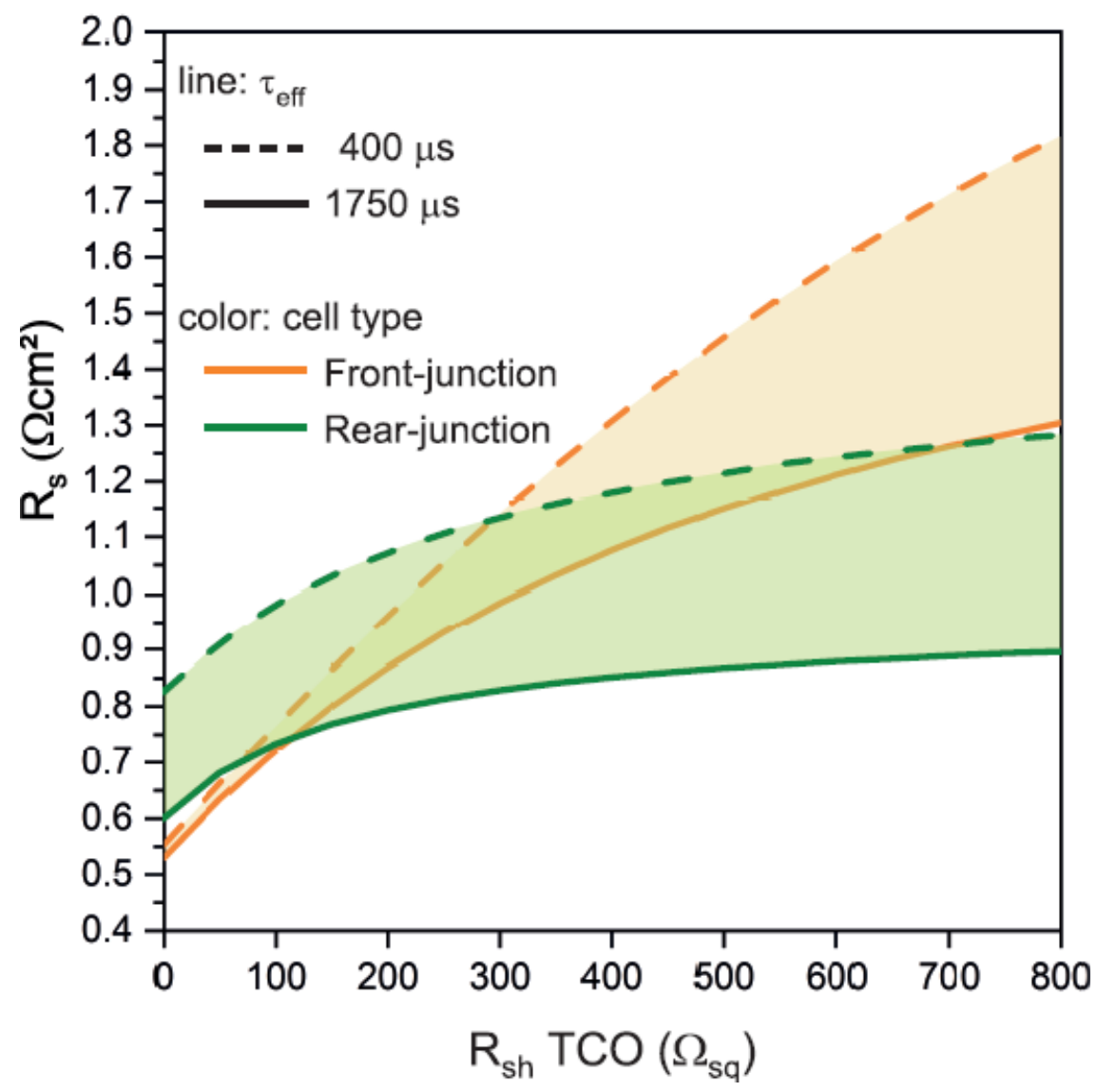


Figure 5. Simulated $R_{s}$ dependence on front-TCO $R_{s h}$ for rear-junction (green lines) and front-junction (orange lines) devices with $400 \mu \mathrm{s}$ (dashed lines) and $1750 \mu$ s (solid lines) $\tau_{e f f}$ lifetime.

\subsubsection{Simulated Solar Cells Parameters}

Based on the simulated values for $J_{\mathrm{sc}}$, reduced by $3 \%\left(\sim 1.2 \mathrm{~mA} / \mathrm{cm}^{2}\right)$ due to the grid shading, and along with $R_{\mathrm{s}}$ obtained from the electrical simulations we can calculate the cell parameters. The open circuit voltage $V_{\text {oc }}$ shows values from 729 to $730 \mathrm{mV}$ slighlty varying with the TCO's $R_{s}$. Table 1 summarizes the simulated values for $R_{s}, F F$ and the efficiency $\eta$ for the "standard" $75 \mathrm{~nm}$ thick TCOs and for the thinner TCOs both with a-SiO${ }_{2}$ capping. When reducing the TCO thicknesses an $R_{s}$ increase and $F F$ decrease results, however the device $\eta$ stays the same or even increases due to the optical performance benefit. We also see that the $\mathrm{ZnO}: \mathrm{Al}$ front-contacted rear-junction device shows a very similar $\eta$ of $22.7 \%$ in comparison to its ITO counterpart with $22.8 \%$. In contrast, for a front-junction solar cell design the $\eta$ of a $\mathrm{ZnO}$ :Al device considerably drops to $22.1 \%$. The IO:H will result in the best efficiency being around $23 \%$ as compared to the other two TCO materials independently of the device configuration due to its higher conductivity.

Considering the grid-finger pitch, that was originally optimized for the cells with ITO, the ZnO:Al will slightly improve its $\eta$ when narrowing the pitch to $1430 \mu \mathrm{m}$ and, oppositely, the IO:H cells by increasing it to $1800 \mu \mathrm{m}$. Nonetheless, we found that this effect, has a small impact on the device efficiency of only $+/-0.1 \%$ (abs), as we simulated the full effect of varying finger pitches at a constant finger width for all TCOs (not shown).

Table 1. Calculated $R_{s h}$ from single layers on glass as well as simulated $R_{s}, F F$ and $\eta$ for standard and optimized front-contact TCO thicknesses. At optimized TCO thickness $R_{s}, F F$ and $\eta$ are shown for a front-emitter cell design simulation for comparison.

\begin{tabular}{|c|c|c|c|c|c|c|c|c|c|c|c|c|c|}
\hline & \multicolumn{4}{|c|}{$\begin{array}{c}\text { Standard TCO thickness } \\
\text { rear-junction }\end{array}$} & \multicolumn{5}{c|}{$\begin{array}{c}\text { Optimized TCO thickness } \\
\text { rear-junction }\end{array}$} \\
\hline TCO & $\begin{array}{c}t \\
(\mathrm{~nm})\end{array}$ & $\begin{array}{c}R_{s h} \\
(\Omega)\end{array}$ & $\begin{array}{c}R_{s} \\
\left(\Omega \mathrm{cm}^{2}\right)\end{array}$ & $\begin{array}{c}F F \\
(\%)\end{array}$ & $\begin{array}{c}\eta \\
(\%)\end{array}$ & $\begin{array}{c}t \\
(\mathrm{~nm})\end{array}$ & $\begin{array}{c}R_{s h} \\
(\Omega)\end{array}$ & $\begin{array}{c}R_{s} \\
\left(\Omega \mathrm{cm}^{2}\right)\end{array}$ & $\begin{array}{c}F F \\
(\%)\end{array}$ & $\begin{array}{c}\eta \\
(\%)\end{array}$ & $\begin{array}{c}F F \\
(\%)\end{array}$ & $\begin{array}{c}R_{s} \\
\left(\Omega \mathrm{cm}^{2}\right)\end{array}$ & $\begin{array}{c}\eta \\
(\%)\end{array}$ \\
\hline ITO & 75 & 100 & 0.74 & 79.8 & 22.6 & 40 & 190 & 0.80 & 79.5 & 22.8 & 78.9 & 0.86 & 22.5 \\
ZnO:Al & 75 & 260 & 0.82 & 79.4 & 22.6 & 40 & 500 & 0.88 & 79.1 & 22.7 & 77.2 & 1.16 & 22.1 \\
IO:H & 75 & 60 & 0.70 & 80.1 & 23.1 & 55 & 80 & 0.72 & 80.0 & 23.1 & 79.8 & 0.69 & 22.9 \\
\hline
\end{tabular}

\subsection{Experimental Solar Cell Results}

We prepared SHJ cells following the optimized and simulated designs as discussed above, namely, for all three TCOs the standard and the optimized thin version, both with a-SiO $\mathrm{S}_{2}$ capping. Figure 6 shows the $J-V$ parameters of these cells. Generally, we observe that the trends in $J_{\text {Generated }}$ resemble the simulated ones. The IO:H clearly outperforms the other two TCOs in terms of current density and the cells with optimized TCO thicknesses generally perform better than the "standard" ones, while the largest benefit is found for the ITO, as expected. For $V_{\mathrm{oc}}$ and $p F F$, however, we observe effects that were not expected and were not considered in the Quokka2 simulations. Firstly, $V_{\text {oc }}$ and $p F F$ are mostly increased for the "optimized" TCO stack as compared to the "standard" one. Secondly, the cells with IO:H suffer $\sim 15 \mathrm{mV} V_{\mathrm{oc}}$ loss and $2 \%$ (abs.) pFF loss as compared to the cells with ITO or ZnO:Al. The first observation might be explained by two effects: (1) the thinner TCO sputtering could result in less sputter damage, or (2) the deposition of the a-SiO 2 layer by PECVD leads to a curing effect due to the reactive hydrogen that is present during PECVD. The hydrogen might penetrate the TCO layer, subsequently, passivating defects at the Si surface. With a thinner TCO this passivation might be more effective due to the shorter penetration path, leading to improved $V_{\mathrm{oc}}$ and $p F F$. The second effect most likely can be ascribed to the IO:H processing, since the samples are thermally annealed in vacuum for one hour after TCO sputtering. Results of lifetime measurements (not shown) of c-Si/i-a-Si:H/p-a-Si:H (i/p) symmetric samples highlight that the thermal budget for this annealing process is excessive for the device, thereby, diminishing the passivation of the 
c-Si wafer. There is a trade-off between the optimal crystallization of the IO:H and the passivation properties of the $\mathrm{i} / \mathrm{p}$ layers after thermal processes. Optimizing this process step and avoiding the $V_{\mathrm{oc}}$ and $p F F$ degradation should lead to the expected best cell efficiency $>23 \%$ for IO: $\mathrm{H}$. The main parameters that can be varied for this purpose are the annealing temperature, time and atmosphere (air, vacuum). We also can discard a strong difference in sputtering damage for the different devices. All devices became the same $\mathrm{ZnO}: \mathrm{Al} / \mathrm{silver}$ back contact at the $\mathrm{i} / \mathrm{p}$ contact side and for the $\mathrm{i} / \mathrm{n}$ passivating layers we have seen that the $i F F$ and $i V_{o c}$ are fully recovered after the respective annealing processes for all TCOs.

In order to test the initial assumption on the benefit of using a rear-junction configuration, as was shown in Figure 5 with the relation between the simulated cell's $R_{\mathrm{s}}$ and the TCO's $R_{\mathrm{sh}}$, we plot experimental values of $R_{s}$ vs. $R_{\mathrm{sh}}$ together with the simulated curves, shown in Figure 7. We found that after a- $\mathrm{SiO}_{2}$ deposition the $R_{s h}$, as deduced from TLM measurements through the method described in section 2.2., is reduced by 20 to $45 \%$ for all three TCOs. For this reason, we see a discrepancy between the calculated values from the layers on glass described in section 3.1.1. when compared to those ones deducted from TLM structures on the wafer. Ritzau et al. [26] reported a similar effect. They observed a lowering of $R_{s h}$ for ITO annealed on thin-film a-Si:H layers. Similar to that, Herasimneka et al. [12] noticed an $R_{s h}$ reduction when depositing a-SiO ${ }_{x}: \mathrm{H}$ on top of ITO layers as second ARC. As was also discussed above, hydrogen radicals, present during deposition of a-SiO${ }_{2}$ by $\mathrm{PECVD}$, might diffuse through and into the TCO improving its electrical properties, e.g. due to grain boundary passivation.
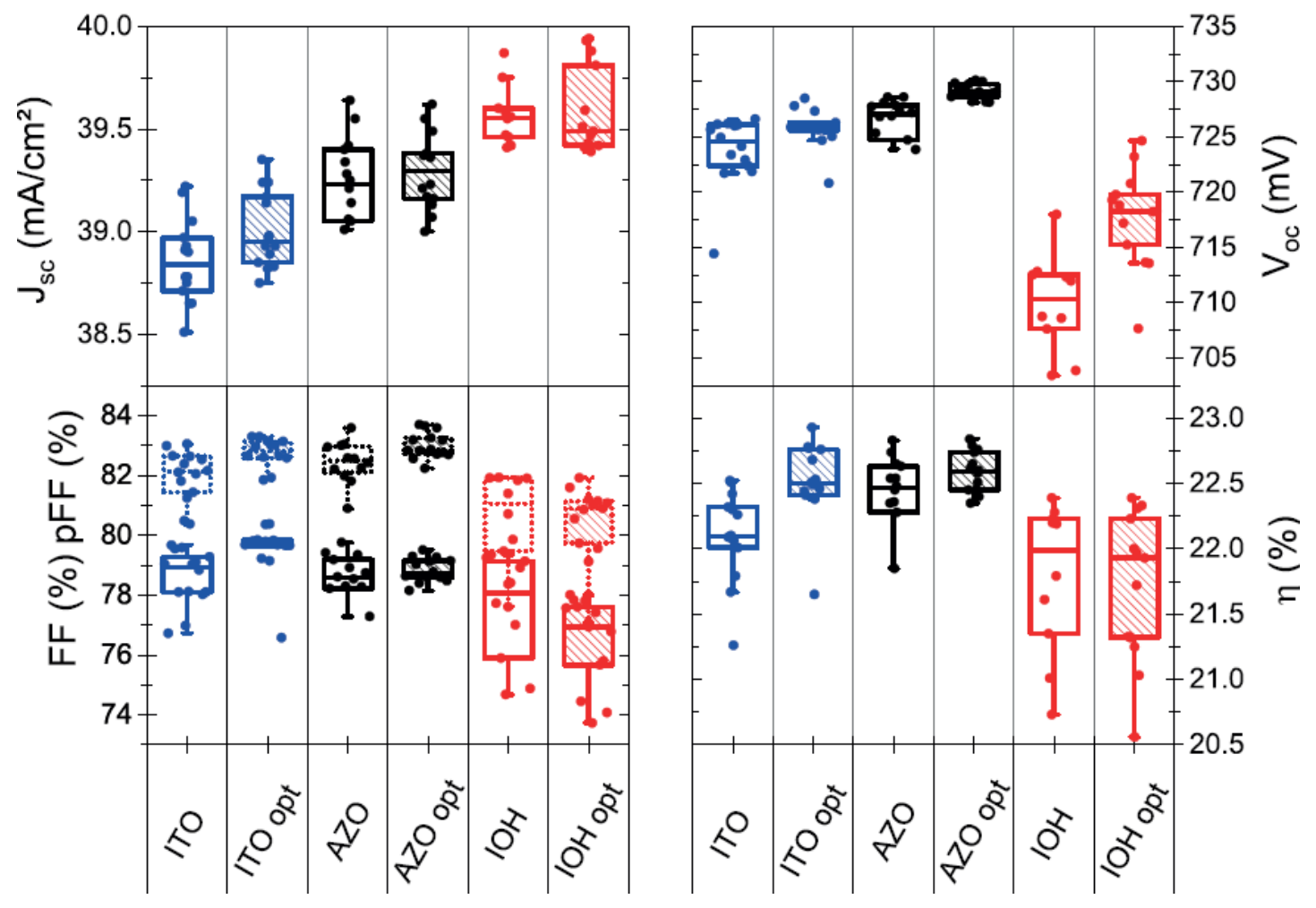

Figure 6. Current-voltage parameters of experimental cells that were prepared with a standard $75 \mathrm{~nm}$ TCO thickness and at their optimized thickness, both with a-SiO 2 capping (compare Figure 4). The pseudo fill-factor is plotted on the fill factor graph with dotted borders. The boxplots are shown with median values indicated.

In Figure 6 we see that even when using ZnO:Al with an $R_{\text {sh }}$ as high as $250 \Omega$ the cell's $R_{\mathrm{s}}$ is increased by only $0.2 \Omega \mathrm{cm}^{2}$ as compared to the ITO reference. Also shown in the graph is the simulated trend for a front junction solar cell and the $R_{\mathrm{s}}$ value for the sample with highest $R_{s h}$ with the thin $\mathrm{ZnO}$ :Al before a- $\mathrm{SiO}_{2}$ capping. We see that in this $R_{\mathrm{sh}}$ range the experimental $R_{\mathrm{s}}$ value is higher than simulated for the rear-junction trend but clearly lower than expected for front-junction cells. This might be an indication of an overestimation of the lateral transport support by the wafer in this range. Moreover, there is uncertainty in the resistance contribution from the TCO/n-layer contact, which was included in the lumped $R_{S}$ simulation value being the same for all samples. Nonetheless, we can confirm that the $R_{s}$ of the cell with $\mathrm{ZnO}: \mathrm{Al}$ remains in a competitive level when comparing to the ITO cell. Concerning the impact of the a-SiOx layer on device stability under hot and humid atmosphere, Adachi et al. investigated the effects of a-SiO $\mathrm{x}_{\mathrm{x}}$ capping on SHJ solar cells a as a barrier on top of ITO transparent electrodes and concluded that 
this layer improves the damp-heat (DH) stability of encapsulated cells [31]. In a recent study we showed there is also a benefit for the stability of $\mathrm{ZnO}$; $\mathrm{Al}$ contacted full-size encapsulated SHJ cells having same good stability as ITO references [32].

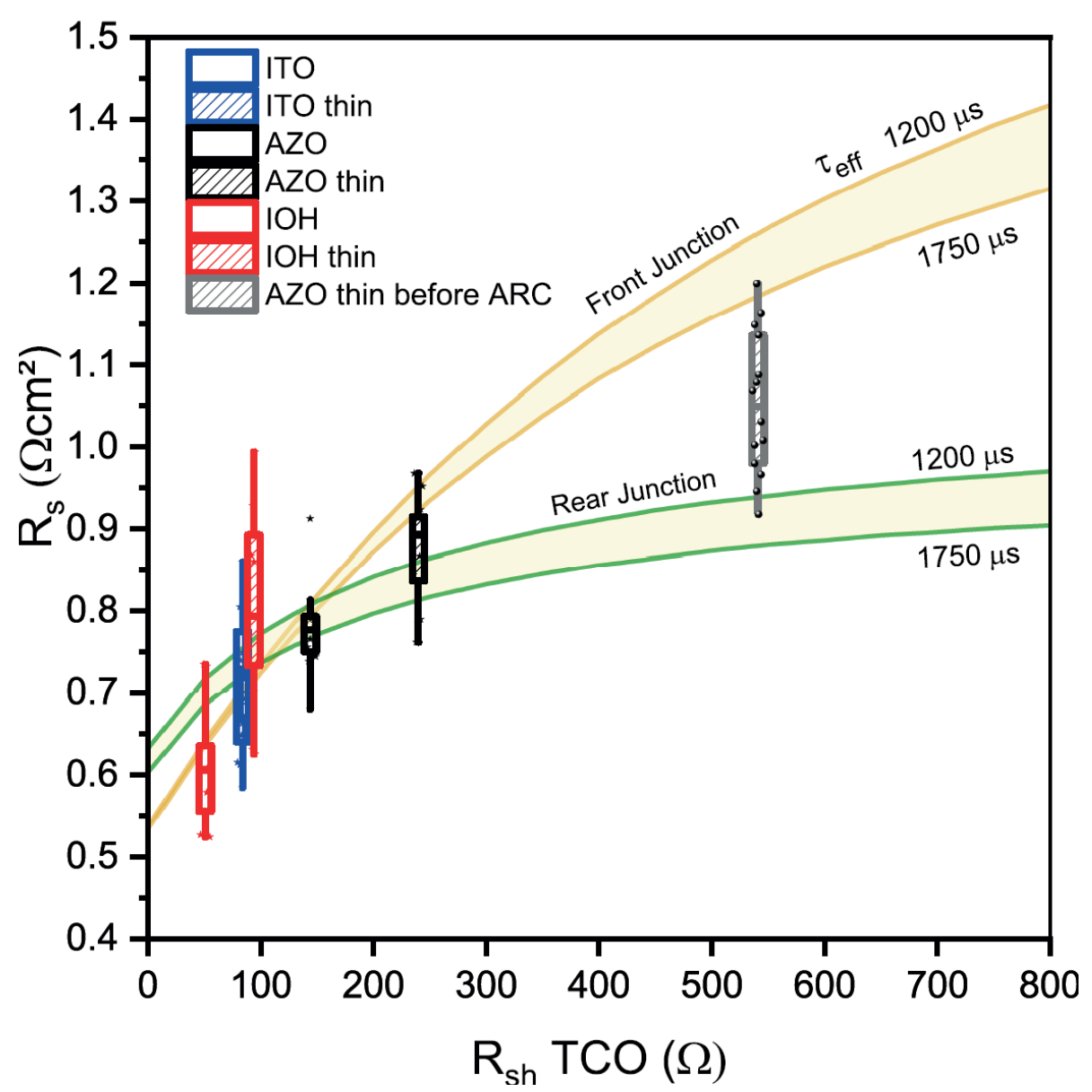

Figure 7. $R_{\mathrm{s}}$ values of experimental solar cells calculated from the dark to light $J-V$ curves comparison as a function of TCO $R_{s h}$ extracted from TLM structures of the same samples. The simulated $R_{s}$ for a $\tau_{\text {eff }}$ ranging from 1200 to $1750 \mu \mathrm{s}$ for rear- and frontjunction SHJ cells are added as a guideline (compare to Figure 5). The boxplots are shown with median values indicated.

\section{Conclusions}

In summary, we demonstrated by optical and electrical simulations as well as with experimental results the effect of different front TCOs on the performance of rear-junction SHJ solar cells. High-mobility TCOs, such as IO:H, are expected to outperform other TCOs under investigation, i.e. ITO and $\mathrm{ZnO}$ :Al, with a predicted efficiency benefit of $0.4 \%$ (abs.). In our experimental cell results, however, degradation of $V_{\mathrm{oc}}$ and $p F F$ overcompensates the optical benefit. We expect to solve this by reducing the thermal budget during IO:H annealing. Experimental devices confirm that optimized thinner TCO layers will result in an 
efficiency enhancement or, in the worst case, allow for the same performance while offering the advantage of cost reduction by TCO material saving. We showed the beneficial optical effect of a PECVD a-SiO 2 capping layer after TCO sputtering, asalso proposed by other groups. Moreover, we found strong indications that this layer can even improve electrical cell properties, i.e. $V_{\mathrm{oc}}$ and $p F F$, in particular for thin TCOs, which we explain by hydrogen passivation of the silicon surface during the PECVD deposition.

The electrical simulations propose that a lesser conductive TCO in the example of $\mathrm{ZnO}$ :Al can be implemented in a rear-junction device without compromising the solar cell's $R_{s}$. Experimental results confirm, that the relatively resistive but very transparent $\mathrm{ZnO}: \mathrm{Al}$ is an attractive alternative to ITO, yielding very similar efficiencies when implemented in rear-junction SHJ solar cells. These results pave the way for the application of the abundant and cost-effective $\mathrm{ZnO}$ :Al substituting indium-based TCOs in SHJ solar cell mass production.

\section{Acknowledgement}

We would like to thank the co-workers at HZB for their support. Katja Mayer-Stillrich and Manuel Hartig for sputtering depositions. Mathias Zelt and Tobias Henschel for support with PECVD. Holger Rhein for screen-printing processing. Darja Erfurt and Stefan Körner for fruitful discussions. Dr. Florian Ruske for support with ellipsometry measurements and optical modelling. This work was partially supported by the German Ministry of Economic Affairs and Energy (BMWi) in the framework of the HERA project (reference \#03258251). Alexandros Cruz gratefully acknowledges the scholarship support from the Mexican National Council for Science and Technology (CONACYT).

Figure 1. Mobility vs carrier concentration of $105 \pm 10 \mathrm{~nm}$ thick ITO, ZnO:Al and IO:H layers on glass. The colored ellipses highlight the range of TCO properties applied on SHJ experimental solar cells. The filled symbols correspond to layers optically analyzed. The sheet resistance is calculated and plotted for $105 \mathrm{~nm}$ TCO thickness.

Figure 2. Optical absorption spectra of selected 105 $\pm 10 \mathrm{~nm}$ ITO, ZnO:Al and IO:H layers on glass and generated current density per wavelength of a standard SHJ solar cell from GenPro4 simulations. The dashed line is for a thinner IO:H 55 \pm 5 nm layer.

Figure 3. Current gain or loss $\triangle J$ in dependence of TCO thickness. The values are referenced to the current absorbed by each TCO at their standard thickness of $75 \mathrm{~nm}$. Current gain values are noted for $40 \mathrm{~nm}$ TCO thickness.

Figure 4. Top row: Colormaps of current density loss due to device reflection $J_{\text {Reflection }}$. Bottom row: Colormaps of current density generated $J_{\text {Generated }}$ in the device. For all graphs the TCO (ITO, $\mathrm{ZnO}: \mathrm{Al}$ and $\mathrm{IO}: \mathrm{H}$ ) and the a-SiO $\mathrm{S}_{2}$ second ARC thickness on simulated silicon heterojunction solar cells are varied. Generated current in the silicon absorber $J_{\text {Generated }}$ values for $75 \mathrm{~nm}$ and optimal TCO thickness with and without a-SiO 2 optimal layers are noted.

Figure 5. Simulated $R_{s}$ dependence on front-TCO $R_{s h}$ for rear-junction (green lines) and front-junction (orange lines) devices with $400 \mu \mathrm{s}$ (dashed lines) and $1750 \mu \mathrm{s}$ (solid lines) $\tau_{\text {eff lifetime. }}$ ling

Figure 6. Current-voltage parameters of experimental cells that were prepared with a standard 75 nm TCO thickness and at their optimized thickness, both with a-SiO 2 capping (compare Figure 4). The pseudo fill-factor is plotted on the fill factor graph as dotted lines. The boxplots are shown with median values indicated.

Figure 7. $R_{\mathrm{S}}$ values of experimental solar cells calculated from the dark to light $J-V$ curves comparison as a function of TCO $R_{s h}$ extracted from TLM structures of the same samples. The simulated $R_{s}$ for a $\tau_{e f f}$ ranging from 1200 to $1750 \mu$ s for rear- and frontjunction SHJ cells are added as a guideline (compare to Figure 5). The boxplots are shown with median values indicated.

\section{REFERENCES}

[1] K. Yoshikawa et al., "Silicon heterojunction solar cell with interdigitated back contacts for a photoconversion efficiency over 26\%," Nat. Energy, vol. 2, no. 5, p. 17032, May 2017. 
[2] D. Adachi, J. L. Hernández, and K. Yamamoto, "Impact of carrier recombination on fill factor for large area heterojunction crystalline silicon solar cell with 25.1\% efficiency," Appl. Phys. Lett., vol. 107, no. 23, p. 233506, Dec. 2015.

[3] M. A. Green et al., "Solar cell efficiency tables (version 50)," Prog. Photovolt. Res. Appl., vol. 25, no. 7, pp. 668-676, Jul. 2017.

[4] J. Haschke, O. Dupré, M. Boccard, and C. Ballif, "Silicon heterojunction solar cells: Recent technological development and practical aspects - from lab to industry," Sol. Energy Mater. Sol. Cells, vol. 187, pp. 140-153, Dec. 2018.

[5] M. Bivour, S. Schröer, M. Hermle, and S. W. Glunz, "Silicon heterojunction rear emitter solar cells: Less restrictions on the optoelectrical properties of front side TCOs," Sol. Energy Mater. Sol. Cells, vol. 122, pp. 120-129, Mar. 2014.

[6] T. Tohsophon, A. Dabirian, S. D. Wolf, M. Morales-Masis, and C. Ballif, "Environmental stability of high-mobility indiumoxide based transparent electrodes," APL Mater., vol. 3, no. 11, p. 116105, Nov. 2015.

[7] T. Minami, "Substitution of transparent conducting oxide thin films for indium tin oxide transparent electrode applications," Thin Solid Films, vol. 516, no. 7, pp. 1314-1321, Feb. 2008.

[8] T. Koida, H. Fujiwara, and M. Kondo, "High-mobility hydrogen-doped transparent conductive oxide for a-Si:H/c-Si heterojunction solar cells,” Sol. Energy Mater. Sol. Cells, vol. 93, no. 6-7, pp. 851-854, Jun. 2009.

[9] Z. C. Holman et al., "Current Losses at the Front of Silicon Heterojunction Solar Cells," IEEE J. Photovolt., vol. 2, no. 1, pp. 7-15, Jan. 2012.

[10] M. Balestrieri, D. Pysch, J.-P. Becker, M. Hermle, W. Warta, and S. W. Glunz, "Characterization and optimization of indium tin oxide films for heterojunction solar cells," Sol. Energy Mater. Sol. Cells, vol. 95, no. 8, pp. $2390-2399$, Aug. 2011.

[11] C. Zhou et al., "SiOx(C)/SiNx dual-layer anti-reflectance film coating for improved cell efficiency," Sol. Energy, vol. 85, no. 11, pp. 3057-3063, Nov. 2011.

[12] S. Y. Herasimenka, W. J. Dauksher, M. Boccard, and S. Bowden, "ITO/SiOx:H stacks for silicon heterojunction solar cells," Sol. Energy Mater. Sol. Cells, vol. 158, Part 1, pp. 98-101, Dec. 2016.

[13] D. Zhang et al., "Design and fabrication of a SiOx/ITO double-layer anti-reflective coating for heterojunction silicon solar cells," Sol. Energy Mater. Sol. Cells, vol. 117, pp. 132-138, Oktober 2013.

[14] J. Geissbühler et al., "Silicon Heterojunction Solar Cells With Copper-Plated Grid Electrodes: Status and Comparison With Silver Thick-Film Techniques," IEEE J. Photovolt., vol. 4, no. 4, pp. 1055-1062, Jul. 2014.

[15] R. Santbergen, A. H. M. Smets, and M. Zeman, "Optical model for multilayer structures with coherent, partly coherent and incoherent layers," Opt. Express, vol. 21, no. 102, pp. A262-A267, Mar. 2013.

[16] A. Fell, "A Free and Fast Three-Dimensional/Two-Dimensional Solar Cell Simulator Featuring Conductive Boundary and Quasi-Neutrality Approximations," IEEE Trans. Electron Devices, vol. 60, no. 2, pp. 733-738, Feb. 2013.

[17] A. Pflug, V. Sittinger, F. Ruske, B. Szyszka, and G. Dittmar, "Optical characterization of aluminum-doped zinc oxide films by advanced dispersion theories," Thin Solid Films, vol. 455-456, pp. 201-206, May 2004.

[18] W. Kern, “The Evolution of Silicon Wafer Cleaning Technology,” J. Electrochem. Soc., vol. 137, no. 6, pp. 1887-1892, Jun. 1990.

[19] A. B. Morales-Vilches et al., "Nanocrystalline vs. amorphous n-type silicon front surface field layers in silicon heterojunction solar cells: Role of thickness and oxygen content.," Proc EUPVSEC 2017.

[20] D. Pysch, A. Mette, and S. W. Glunz, "A review and comparison of different methods to determine the series resistance of solar cells," Sol. Energy Mater. Sol. Cells, vol. 91, no. 18, pp. 1698-1706, Nov. 2007.

[21] A. Valla, P. Carroy, F. Ozanne, and D. Muñoz, "Understanding the role of mobility of ITO films for silicon heterojunction solar cell applications," Sol. Energy Mater. Sol. Cells, vol. 157, pp. 874-880, Dec. 2016.

[22] K. Ellmer, F. Kudella, R. Mientus, R. Schieck, and S. Fiechter, "Influence of discharge parameters on the layer properties of reactive magnetron sputtered ZnO:Al films," Thin Solid Films, vol. 247, no. 1, pp. 15-23, Jul. 1994.

[23] T. Koida, M. Kondo, K. Tsutsumi, A. Sakaguchi, M. Suzuki, and H. Fujiwara, "Hydrogen-doped In2O3 transparent conducting oxide films prepared by solid-phase crystallization method," J. Appl. Phys., vol. 107, no. 3, p. 33514, Feb. 2010.

[24] V. Srikant and D. R. Clarke, "Optical absorption edge of ZnO thin films: The effect of substrate," J. Appl. Phys., vol. 81, no. 9, pp. 6357-6364, May 1997.

[25] T. Koida, H. Fujiwara, and M. Kondo, "Reduction of Optical Loss in Hydrogenated Amorphous Silicon/Crystalline Silicon Heterojunction Solar Cells by High-Mobility Hydrogen-Doped $\mathrm{In}_{2} \mathrm{O}_{3}$ Transparent Conductive Oxide," Appl. Phys. Express, vol. 1, p. 41501, Mar. 2008.

[26] K.-U. Ritzau, T. Behrendt, D. Palaferri, M. Bivour, and M. Hermle, "Hydrogen doping of Indium Tin Oxide due to thermal treatment of hetero-junction solar cells,” Thin Solid Films, vol. 599, no. Supplement C, pp. 161-165, Jan. 2016.

[27] D. B. M. Klaassen, "A unified mobility model for device simulation-I. Model equations and concentration dependence," Solid-State Electron., vol. 35, no. 7, pp. 953-959, Jul. 1992.

[28] A. Richter, S. W. Glunz, F. Werner, J. Schmidt, and A. Cuevas, "Improved quantitative description of Auger recombination in crystalline silicon," Phys. Rev. B, vol. 86, no. 16, p. 165202, Oct. 2012.

[29] T. Trupke et al., "Temperature dependence of the radiative recombination coefficient of intrinsic crystalline silicon," J. Appl. Phys., vol. 94, no. 8, pp. 4930-4937, Sep. 2003. 
[30] H. H. Berger, “Models for contacts to planar devices," Solid-State Electron., vol. 15, no. 2, pp. 145-158, Feb. 1972.

[31] D. Adachi, "Effects of SiOx barrier layer prepared by plasma-enhanced chemical vapor deposition on improvement of longterm reliability and production cost for Cu-plated amorphous Si/crystalline Si heterojunction solar cells.".

[32] A. B. Morales-Vilches et al., "ITO-Free Silicon Heterojunction Solar Cells With ZnO:Al/SiO2 Front Electrodes Reaching a Conversion Efficiency of 23\%," IEEE J. Photovolt., pp. 1-6, 2018. 